\title{
Pexophagy in yeast and mammals: an update on mysteries
}

\section{Review Article}

\section{Author(s):}

Eberhart, Tanja; Kovacs, Werner J.

Publication date:

2018-11

\section{Permanent link:}

https://doi.org/10.3929/ethz-b-000302080

Rights / license:

In Copyright - Non-Commercial Use Permitted

Originally published in:

Histochemistry and Cell Biology 150(5), https://doi.org/10.1007/s00418-018-1724-3

Funding acknowledgement:

166245 - Exploring the molecular mechanism of Hif-2alpha-mediated pexophagy and the role of pexophagy in tumorigenesis (SNF) 


\title{
Pexophagy in yeast and mammals: an update on mysteries
}

\author{
Tanja Eberhart, Werner J. Kovacs* \\ Institute of Molecular Health Sciences, ETH Zurich, Zurich, Switzerland. \\ *Correspondence to: Werner Kovacs, Institute of Molecular Health Sciences, ETH Zurich, Otto-Stern- \\ Weg 7, HPL H16, CH-8093 Zurich, Switzerland. Tel.: +41 446333084. \\ E-mail: werner.kovacs@biol.ethz.ch
}




\section{Keywords}

Peroxisomes, autophagy, selective autophagy, pexophagy, yeast, mammalian, organelle. 


\section{Introduction}

Peroxisomes are extremely versatile and dynamic organelles whose number, size, and function are dependent on cell type and metabolic needs. They play key roles in the degradation of fatty acids (i.e., very long-chain, branched-chain, and polyunsaturated fatty acids), ether lipid synthesis, cholesterol and bile acid synthesis, and metabolism of reactive oxygen species (ROS) (Faust and Kovacs 2014; Fransen et al. 2012; Schönenberger and Kovacs 2015; Van Veldhoven 2010). They also act as intracellular signaling platforms in redox, lipid, inflammatory, and innate immune signaling (Dixit et al. 2010; Dorninger et al. 2015; Lodhi et al. 2015a; Lodhi et al. 2015b; Nordgren and Fransen 2014; Odendall et al. 2014). The importance of peroxisomal metabolism is illustrated by the marked abnormalities in brain and systemic organs in peroxisome biogenesis disorders of the Zellweger spectrum in which functional peroxisomes are absent or disorders caused by single peroxisomal enzyme deficiencies (Raymond et al. 2009). These multisystemic diseases manifest specific clinical and biochemical disturbances that originate from the affected peroxisomal pathways. Recent studies support a peroxisomal role in the pathogenesis of nonalcoholic fatty liver disease and steatohepatitis, aging, diabetes, and neurodegenerative disorders (Cipolla and Lodhi 2017; Fransen et al. 2013; Puri et al. 2007; Puri et al. 2009). A decrease in peroxisome abundance has been observed in various tumor cells (Schönenberger and Kovacs 2015), and at least in human clear cell renal cell carcinomas (ccRCC) the abundance of peroxisomes is decreased by their enhanced degradation via selective autophagy (Walter et al. 2014).

Peroxisome homeostasis is achieved by balancing biogenesis and degradation of peroxisomes in response to changing environmental conditions. Three mechanisms for peroxisome degradation have been described in mammalian cells, which include selective autophagy (pexophagy), proteolysis by peroxisomal Lon protease 2 (LONP2), and 15-lipoxygenase-1 (ALOX15)-mediated autolysis (Schrader and Fahimi 2008; Yokota and Dariush Fahimi 2009). Studies using liver-specific Atg7 knockout mice revealed that 70$80 \%$ of liver peroxisomes are degraded via pexophagy, while the remaining 20-30\% are degraded by LONP2 and ALOX15 (Iwata et al. 2006; Yokota and Dariush Fahimi 2009). Pexophagy is critical for the maintenance of cellular homeostasis by maintaining both organelle integrity and number in the context of 
varying environments and stresses. Peroxisomes generate reactive oxygen and nitrogen species as byproducts of their oxidative type of metabolism, and removal of damaged organelles is essential to keep a balanced cellular redox state (Walker et al. 2018).

Autophagy is an evolutionary conserved catabolic process for degradation of macromolecules and organelles. Many different signal transduction events can converge to initiate autophagy in response to various stressors. The autophagy process can be divided into at least six sequential steps: (1) initiation, (2) membrane nucleation and phagophore formation, (3) phagophore elongation with concomitant cargo sequestration, (4) phagophore closure forming an autophagosome, a double membrane vesicle, (5) fusion of the autophagosome with the lysosome forming an autolysosome, and (6) degradation of sequestered cargo in the autolysosome (Galluzzi et al. 2017; Mizushima et al. 2011) (Fig. 1a). Autophagosome formation and maturation is a highly regulated process, and key components in this process are the autophagy-related (ATG) proteins (Mercer et al. 2018; Mizushima et al. 2011). Initiation of autophagosome formation is regulated by the UNC-51-like kinase $1 / 2$ (ULK1/2, the mammalian orthologs of Atg1) and class III phosphatidylinositol 3-kinase (PI3K) complexes. The ULK complex consists of ULK1/2, ATG13, FIP200 (Atg17), and ATG101 and translocates upon activation to subdomains of the endoplasmic reticulum (ER), called omegasomes, to drive the nucleation of autophagosomes. The ULK complex activates the class III PI3K complex (PI3K complex 1), which consists of the lipid kinase VPS34 (PIK3C3), Beclin1 (ATG6), ATG14, and the PI3K regulatory subunit 4 (PIK3R4; pseudokinase p150), leading to its translocation to the ER, where it produces phosphatidylinositol 3-phosphate to drive omegasome formation. Phagophore elongation is facilitated by transient interactions with the ATG9 compartment, which delivers lipids for membrane formation from various sources including the ER, recycling endosomes, plasma membrane, mitochondria, and Golgi (Mercer et al. 2018). Elongation and closure of the phagophore membrane is controlled by two ubiquitin-like conjugation pathways that conjugate ATG12 to ATG5 and ATG8 [the microtubule-associated proteins-1 light chain 3 (MAP1LC3) and gamma-aminobutyric acid receptorassociated protein (GABARAP) families are the mammalian ATG8 homologs; for simplicity referred to below as LC3 proteins] to the lipid phosphatidylethanolamine (PE) (Klionsky and Schulman 2014; Noda 
and Inagaki 2015). Briefly, the ubiquitin-like ATG12 is activated by ATG7, an E1-like enzyme, it is then transferred to ATG10, an E2-like enzyme, and it is finally conjugated to ATG5. The ATG12-ATG5 conjugate forms a complex with ATG16 and is recruited to the phagophore. The main role of the ATG12ATG5-ATG16 complex is E3-like activity for the other autophagy-specific ubiquitin-like system, LC3. LC3 proteins are synthesized as precursor proteins and are predominantly found in an unlipidated form in the cytosol, referred to as LC3-I. Prior to lipidation the LC3 precursor is proteolytically processed by ATG4. The processed LC3 is conjugated to PE by ATG7, the E2-like enzyme ATG3, and the ATG12-ATG5ATG16 complex, forming the lipidated LC3-II. LC3-II localizes to both outer and inner membranes of phagophores. The ATG12-ATG5-ATG16 complex leaves the autophagosome during its maturation, and the LC3-II associated with the autophagosomal cytosolic surfaces is cleaved from the PE by ATG4 and recycled. The regulation of LC3 lipidation and its association with membranes to perform its autophagy function is driven by the PI3P production by the PI3K complex 1 . The autophagosome fuses with endocytic and lysosomal compartments to form an autolysosome (Yu et al. 2018). The inner membrane of the autolysosome is lysed and the cargos are degraded through the activity of lysosomal hydrolases. The degradation products are transported out of the autolysosome into the cytosol for reuse. Once the degradation of autophagy cargos is completed, autolysosomes disintegrate and contribute to the regeneration of the lysosomal pool via a process termed autophagic lysosome reformation (Yu et al. 2010).

Both non-selective macroautophagy and selective autophagy have been described (Johansen and Lamark 2011; Mizushima et al. 2011; Schreiber and Peter 2014). Most selective autophagy pathways use a common mechanism, including the core autophagic machinery and a set of selectivity factors. Important among the selectivity factors are selective autophagy receptors which mark each specific cargo for selective degradation and connect the cargo to the core autophagic machinery via Atg8-interacting motifs (AIM) and LC3-interacting regions (LIR) to Atg8 and the mammalian homologs of Atg8, respectively (Birgisdottir et al. 2013; Khaminets et al. 2016; Mancias and Kimmelman 2016) (Fig. 1b). Additional posttranslational modifications can enhance substrate recognition and selectivity (Khaminets et al. 2016; McEwan and Dikic 2011). 


\section{Pexophagy in yeasts}

The molecular machineries of pexophagy have been elucidated from studies of the budding yeast Saccharomyces cerevisiae and the methylotrophic yeasts Pichia pastoris and Hansenula polymorpha (Fig. 2a). Incubating these yeasts in oleic acid or methanol leads to peroxisome proliferation, and pexophagy is rapidly induced when peroxisomal metabolism is no longer critical for viability and cell growth following a shift to a preferred carbon source such as glucose (Tuttle and Dunn 1995). There are two main modes of pexophagy in yeast, i.e. macropexophagy and micropexophagy (pexophagy through a microautophagic process). Macropexophagy involves three characteristic steps: recognition of the peroxisome destined for degradation, formation of the pexophagosome, and fusion with the vacuole and degradation of the cargo by vacuolar hydrolases. During selective autophagy formation of the phagophore assembly site (PAS) is mediated by the interaction of activated cargo-bound selective autophagy receptors with the core proteins of the autophagic machinery (Farre and Subramani 2016).

In $P$. pastoris macropexophagy occurs when the carbon source is switched from methanol to ethanol or nitrogen-depleted starvation medium (Farre et al. 2008). In H. polymorpha transfer from methanol to either glucose- or ethanol-containing medium induces macropexophagy (van Zutphen et al. 2008). In S. cerevisiae macropexophagy occurs under conditions of postlogarithmic respiratory growth or after proliferation of peroxisomes in oleate-containing medium followed by glucose-rich, nitrogen-limiting conditions (Hutchins et al. 1999; Motley et al. 2012b). The pexophagy receptors Atg30 and Atg36 were identified in P. pastoris and S. cerevisiae, respectively, and their overexpression stimulates pexophagy even under peroxisome-inducing conditions (Farre et al. 2008; Motley et al. 2012b) (Fig. 2a). Atg30 and Atg36 are associated with peroxisomes during their biogenesis, long before pexophagy induction. These two receptors are not structural homologs, but they share high functional similarity and both the AIM and Atg11-binding regions (A11BRs) are conserved (Oku and Sakai 2016). Atg11 is an essential protein for most selective autophagy pathways in yeast and acts as a scaffold protein in assembling the PAS by interacting with the selective autophagy receptors, with itself, the Atg17 scaffold complex and the Atg1 kinase complex (Farre and Subramani 2016). Atg30 binds to the peroxins Pex3 and Pex 14 as well as the 

(Fig. 2a). Atg30 interacts with the integral peroxisomal membrane protein Atg37, an acyl-CoA-binding protein that interacts with palmitoyl-CoA (Nazarko 2014; Nazarko et al. 2014). The C-terminal transmembrane domain of Atg37 anchors the protein in the peroxisomal membrane and the N-terminal acyl-CoA binding domain is exposed to the cytosol. Atg37 cannot be classified as a bona fide pexophagy receptor, because it does not interact with Atg8 and overexpression of Atg37 neither induces nor interferes with pexophagy. A recent study showed that Atg37 and Pex3 depend on each other for their correct localization at the peroxisomal membrane (Zientara-Rytter et al. 2018). Atg37 is recruited to peroxisomes in a Pex3-dependent manner, and conversely the correct localization of Pex3 on the peroxisomal surface depends on Atg37. Atg30 recruits Atg37 to the pexophagic RPC, where Atg37 regulates the binding of Atg11 to Atg30 (Nazarko et al. 2014). In the absence of Atg37, Atg30 is able to recognize Pex3 and to recruit Atg17, but is unable to recruit Atg11. In vitro experiments have suggested that palmitoyl-CoA and Atg30 compete for the same binding region in Atg37, but palmitoyl-CoA binding to Atg37 does not interfere with Pex3 binding (Nazarko et al. 2014). However, the role of palmitoyl-CoA in pexophagy has not been elucidated in detail. The presence of sufficient local concentrations of palmitoyl-CoA might prevent the activation of pexophagy by inhibiting the interaction between Atg37 and Atg30. The inhibition of the Atg37-Atg30 interaction might be alleviated at low palmitoyl-CoA concentrations due to decreased peroxisomal fatty acid oxidation, thereby allowing Atg30 phosphorylation and pexophagy. A recent study showed that Atg30 requires both Atg37 and Pex3 to recruit Atg8 and Atg11 to the pexophagic RPC (Zientara-Rytter et al. 2018). Atg30 has one binding site for Atg37 and two for Pex3. However, the binding of Atg37 and Pex3 to Atg30 is mutually exclusive due to the close proximity of Atg37- and Pex3-binding sites in the middle domain of $\operatorname{Atg} 30$.

In S. cerevisiae pexophagy relies on the receptor Atg36 that is bound to peroxisomes via the Cterminal cytosolic domain of Pex3 (Motley et al. 2012b) (Fig. 2a). Atg36 also interacts with Atg8 and Atg11 upon induction of pexophagy (Farre et al. 2013; Motley et al. 2012b). The activation of pexophagy in $S$. cerevisiae is a two-step process. Peroxisome-bound Atg36 levels increase under peroxisome proliferation 
conditions, but pexophagy occurs only after Atg36 modification when cells are switched to nitrogen starvation (Motley et al. 2012a).

In yeast, the key regulatory step in selective autophagy appears not to be cargo binding per se, but rather selective autophagy receptor phosphorylation in response to appropriate stimuli engages the autophagic machinery (Farre and Subramani 2016). Indeed, Atg30 is highly phosphorylated as a trigger for pexophagy. It is differentially phosphorylated at S71 and S112 to regulate Atg8 and Atg11 binding, respectively (Farre et al. 2013; Zientara-Rytter et al. 2018). Atg30 is hypophosphorylated in Pex3-deficient cells (Farre et al. 2008) and a recent study identified a region in Pex3 that is required for interaction with Atg30 (Burnett et al. 2015). Mutations in this region do not affect peroxisome biogenesis and peroxisomal localization of Atg30, but cause hypophosphorylation of Atg30 and defects in the interaction of Atg30 with Atg11 as well as impaired recruitment of Atg11 to the pexophagic RPC. In addition, Atg37 is required for proper Atg30 phosphorylation, and an Atg30 mutant which cannot bind Atg37 was weakly phosphorylated, severely affected in its rate of pexophagy and impaired in the recruitment of Atg11 (Nazarko et al. 2014; Zientara-Rytter et al. 2018). Recently, it has been shown that the Hrr25 kinase, a casein kinase $1 \delta$ (CSNK1D) homologue, phosphorylates Atg30 at S112 in order to facilitate interaction with Atg11 (Fig. 2a). Pex3 directly bound to the middle domain of Atg30 negatively regulates its interaction with Hrr25, whereas Atg37 facilitates it (Zientara-Rytter et al. 2018). It has been suggested that this Pex3-Atg30 interaction might prevent premature initiation of pexophagy, but it might also be required to terminate pexophagy (Zientara-Rytter et al. 2018). The recruitment of Atg37 to the pexophagic RPC displaces Pex3 and relieves the inhibition of Hrr25 recruitment to Atg30 by Pex3, thereby allowing Atg30 phosphorylation at S112 and Atg11 recruitment. Pex3 rebinding to the middle domain of Atg30 at a later stage of pexophagy may be a prerequisite for Hrr25 dissociation from the RPC. Since Atg8 and Atg11 cannot interact simultaneously with Atg30 (Farre et al. 2013), Pex3 rebinding might allow further Atg30 modulations such as phosphorylation/dephosphorylation events leading to Atg8 recruitment. It is still unknown what kinase phosphorylates S71 in the Atg8-binding site of Atg30 and how recruitment of this kinase is regulated. 
Atg36 is phosphorylated under both peroxisome proliferation and pexophagy-inducing conditions, but phosphorylation appears to increase upon pexophagy induction, leading to an increased interaction of Atg36 with Atg11 and Atg8 (Motley et al. 2012b). In addition, phosphatase treatment does not result in the same mobility of Atg36 under both conditions. This suggests that other posttranslational modifications apart from phosphorylation might play a role in the induction of Atg36-mediated pexophagy. Hrr25 kinase regulates Atg11 binding to Atg36 by phosphorylating Atg36 at S97 that corresponds to the S112 in Atg30 (Tanaka et al. 2014) (Fig. 2a). Knockdown of Hrr25 diminishes Atg36 phosphorylation and impairs the interaction of Atg36 with Atg11 without affecting its binding to Pex3.

The loss of the peroxisomal AAA ATPase complex components Pex1, Pex6, and Pex15 in $S$. cerevisiae results in enhanced pexophagy and an accumulation of ubiquitinated Pex 5 at the peroxisomal membrane (Nuttall et al. 2014) (Fig. 2a). Whereas ubiquitination is important in selective autophagy in mammalian cells, cargo ubiquitination appears not to play a role in selective autophagy in yeast (Kirkin et al. 2009; Schreiber and Peter 2014; Shaid et al. 2013). The Pex5 receptor cycle is regulated by Pex5 ubiquitination and deubiquitination, but ubiquitinated Pex5 is degraded by the proteasome and not in the vacuole in wild-type yeast (Kiel et al. 2005). Indeed, accumulation of ubiquitinated Pex5 is not the signal for pexophagy in Pexl-deficient cells. Pexl-and Pex6-deficient cells have multiple peroxisomal structures when pexophagy is blocked, indicating that the low number of peroxisomal membranes results from decreased stability and not a defect in peroxisomal membrane formation (Nuttall et al. 2014). Degradation depends on Atg11 and Atg36, which does not bind ubiquitin, and pexophagy in Pexl-deficient cells is blocked when the Atg11-binding motif of Atg36 is mutated. Atg36 is differently modified in Pexl-deficient cells compared with wild-type cells even when its Atg11 binding is prevented. This suggests that Atg36 modification is an early event in pexophagy in Pexl-deficient cells and precedes Atg11 binding. Interestingly, pexophagy is unaffected when the AIM of Atg36 is mutated in Pexl-deficient cells (Nuttall et al. 2014).

In $H$. polymorpha, Pex3 is removed from the peroxisomal surface by the ubiquitin-proteasome system (UPS) prior to sequestration and degradation of the organelle by pexophagy (Bellu et al. 2002; 

lacking the E3 ligases Pex2 and Pex10 (Williams and van der Klei 2013). Moreover, in H. polymorpha it was shown that peroxisomes that are damaged by the abrupt removal of Pex 3 are massively and rapidly degraded by pexophagy even when the cells are placed in conditions that would require peroxisome biogenesis for cell growth (van Zutphen et al. 2011). It is unclear if a similar requirement of Pex3 removal from the peroxisome membrane for pexophagy exists in other methylotrophic yeasts such as $P$. pastoris, where Pex3 is essential to recruit the pexophagy receptor Atg30 to the peroxisome. The peroxin Pex14 also plays an important role in macropexophagy in H. polymorpha (Zutphen et al. 2008). Pex14, specifically its $\mathrm{N}$-terminal region, is required for recognition of the peroxisome by the macropexophagy machinery (Bellu et al. 2001a). However, experimental evidence for an AIM in Pex14 is missing to date. Degradation of peroxisomal membranes ("ghosts") is prevented in cells lacking Pex14, while in other Pex mutant cells peroxisomal membrane structures are normally degraded (Bellu et al. 2001a; Veenhuis et al. 1996). Studies have shown that Pex3 connects the docking site for receptor/cargo protein complexes (Pex13, Pex14, and Pex17) and the RING subcomplex of the peroxisomal importomer (Pex2, Pex10, Pex12) (Hazra et al. 2002). Removal of Pex3 at the onset of pexophagy will disrupt the complex and might lead to a block of further matrix protein import in the peroxisomes that are destined for degradation. It has been hypothesized that the dissociation of the supercomplex might result in exposure of the N-terminal domain of Pex14 and its recognition by a so-far unknown receptor protein to tag the peroxisome for sequestration by the autophagosome (Leao and Kiel 2003; Monastyrska and Klionsky 2006).

A challenging factor for the efficiency of engulfment by the phagophore is organelle size, implying a potential role for fission in the degradation process of both mitochondria and peroxisomes. When mitophagy is induced in S. cerevisiae, the Atg11 scaffold is recruited by the mitophagy receptor Atg 32 , and in turn the dynamin-related GTPase dynamin 1 (Dnm1) is recruited to the degrading mitochondria through its interaction with Atg11 (Mao et al. 2013). The mitochondrial fission complex [Dnm1, mitochondrial fission 1 protein (Fis1), mitochondrial division protein 1 (Mdv1), and CCR4-associated factor 4 (Caf4)] also controls the fission of peroxisomes in yeast (Schrader et al. 2012), and recent findings in S. cerevisiae 


\section{Micropexophagy in yeasts}

In $P$. pastoris another mode of pexophagy, termed micropexophagy, has been demonstrated. Micropexophagy is induced when methanol-grown cells are transferred to glucose medium and involves direct engulfment of the peroxisome by the vacuolar membrane. A planar autophagic membrane structure called the micropexophagy-specific apparatus (MIPA) emerges on the peroxisome surface and fuses with a protrusion of the vacuolar membrane, thereby engulfing the target peroxisome cluster. Finally, membrane scission occurs on the inner side of the vacuolar membrane followed by lysis of the peroxisomes. The choice between induction of either micro- or macropexophagy is determined by ATP levels in the cell (Ano et al. 2005). Micropexophagy was found to be more sensitive to ATP depletion than macropexophagy. This suggests that the micropexophagic process requires a higher level of ATP than macropexophagy and probably reflects an energy-demanding property of vacuolar dynamics. Similar dynamics of vacuole engulfment of the peroxisome were also detected in S. cerevisiae when oleate-grown cells were transferred to glucose medium (Chiang et al. 1996). Microautophagy in H. polymorpha is induced when methanolgrown cells are exposed to nitrogen starvation conditions (Bellu et al. 2001b). However, this process is not considered a selective pathway as cytosolic components are taken up concomitantly with peroxisomes. Interestingly, it was shown that simultaneous treatment of methanol-grown $H$. polymorpha with both 
nitrogen depletion and excess glucose conditions results in concomitant induction of both microautophagy and macropexophagy (Monastryska et al. 2004).

\section{Pexophagy in mammalian cells}

The estimated half-life of mammalian peroxisomes is approximately 1.5-2 days, suggesting that biogenesis and degradation of peroxisomes are dynamic processes (Huybrechts et al. 2009; Poole et al. 1969). Little was known about the regulation of peroxisome degradation in mammalian cells, but recently several studies uncovered mechanisms for regulating mammalian pexophagy. There are no orthologous genes of Atg30 and Atg36 in mammals, but overexpression of neighbor of BRCA1 gene 1 (NBRl) and sequestosome 1 (SQSTM1 or p62), which are autophagy receptors of ubiquitinated targets (Mancias and Kimmelman 2016), induces clustering and degradation of peroxisomes in cell lines (Deosaran et al. 2013). These autophagy receptors contain both an LC3-interacting region that binds to LC3 associated with the phagophore, and a ubiquitin-associated domain that binds to ubiquitinated residues in the target (Kirkin et al. 2009). SQSTM1 is not required for pexophagy when NBR1 is in excess, but its binding to NBR1 increases the efficiency of NBR1-mediated pexophagy (Deosaran et al. 2013). Overexpression of peroxisomal membrane proteins (PMP) (e.g., PMP34 and PEX3) that are fused with a single ubiquitin moiety in their cytosolic domains triggers pexophagy in mammalian cells in a SQSTM1-dependent manner (Kim et al. 2008). However, it is still unknown if a PMP is ubiquitinated under pexophagy-inducing conditions and whether subsequent interaction with NBR1 and/or SQSTM1 links ubiquitinated peroxisomes to the autophagic machinery.

Overexpression of Pex3 in Chinese hamster ovary $(\mathrm{CHO})$ cells and mouse embryonic fibroblasts induced ubiquitination and clustering of peroxisomes and subsequently NBR1-mediated pexophagy, albeit no direct interaction between PEX3 and NBR1 could be detected (Yamashita et al. 2014). However, expression of a Pex3 mutant which was defective in PEX3 ubiquitination did not prevent pexophagy (Yamashita et al. 2014), suggesting that ubiquitination of PEX3 is dispensable for pexophagy and that an endogenous, unidentified peroxisomal protein is ubiquitinated on the peroxisomal membrane and might function in NBR1 recruitment. 
It has been reported that PEX14 is involved in pexophagy in $\mathrm{CHO}$ cells under nutrient starvation by interacting with LC3-II, the lipidated form of LC3 (Hara-Kuge and Fujiki 2008) (Fig. 2b). Cell-free synthesized LC3-II interacts with the transmembrane domain of recombinant PEX14 in an in vitro assay, although PEX14 does not contain a LIR sequence that could ensure LC3 binding (Jiang et al. 2015). It has been proposed that the PEX14-LC3 and PEX14-PEX5 interactions are mutually exclusive and that this competitive interaction might ensure functional segregation of metabolically active and degradation-prone peroxisomes (Hara-Kuge and Fujiki 2008; Jiang et al. 2015). In addition, NBR1 targets to peroxisomes under starvation and it interacts with PEX14 and SQSTM1 (Jiang et al. 2015). Decreased expression levels of NBR1 led to less LC3-II recruitment to peroxisomes, suggesting that NBR1 has a positive effect on the interaction between PEX14 and LC3. Therefore, it has been proposed that NBR1 and/or SQSTM1 are initially targeted to peroxisomes under starvation, thereby inducing a conformational change of PEX14 and exposing its transmembrane domain to enable LC3-II binding (Jiang et al. 2015). Nevertheless, in vivo recruitment of NBR1 and SQSTM1 to peroxisomes and their interaction with PEX14 might also lead to LC3-interaction with their LIR motifs.

Next, we will discuss recent studies that have focused on PEX5 ubiquitination to elucidate mechanisms of pexophagy regulation under certain conditions such as amino acid starvation, oxidative stress, and defects in the peroxisomal matrix protein machinery.

It has been reported that export-deficient mono-ubiquitinated PEX5, generated by PEX5 fusion to a bulky C-terminal green fluorescent protein and mono-ubiquitinated at C11, triggers pexophagy in SV40 large $\mathrm{T}$ antigen-transformed mouse embryonic fibroblasts (Nordgren et al. 2015). However, NBR1 and SQSTM1 were not required for PEX5-GFP-induced pexophagy and this fusion protein did not trigger pexophagy in other cell types. The authors suggested that mono-ubiquitinated PEX5 might serve as a quality control mechanism to remove import-defective peroxisomes.

Peroxisomes are important sites of ROS production and degradation (Schrader and Fahimi 2006). Tight interactions between ROS and autophagy are reflected in two aspects, namely the induction of autophagy by oxidative stress and the reduction of ROS by autophagy. A study reported that the mechanistic target of 
rapamycin complex 1 (mTORC1) regulators tuberous sclerosis complex 1 (TSC1), TSC2, and RHEB (Ras homolog enriched in brain) reside at the cytosolic surface of peroxisomes (Fig. 2b). Activation of the TSC tumor suppressor by peroxisomal ROS repressed mTORC1 and activated autophagy (Zhang et al. 2013). TSC2 and Rheb localization to peroxisomes was lost in peroxisome-deficient human Zellweger cells lacking PEX5-mediated protein import, whereas TSC1 localized to peroxisomal ghosts in Zellweger cells in a putative PEX19-dependent manner. Recently, it has been shown that ataxia-telangiectasia mutated (ATM) kinase is recruited to the cytosolic face of the peroxisomal membrane in a PEX5-dependent manner in response to oxidative stress (Zhang et al. 2015) (Fig. 2b). Activation of cytoplasmic ATM by ROS via the serine/threonine kinase 11 (LKB1) and the AMP-activated protein kinase (AMPK) activates TSC2 to suppress mTORC1 and to induce autophagy (Alexander et al. 2010; Zhang et al. 2015). However, in addition to increasing autophagic flux, ATM also plays a role in pexophagy. Peroxisome-localized ATM phosphorylates PEX5 at S141, which promotes mono-ubiquitination of PEX5 at K209 by the peroxisomal E3-ligases PEX2/10/12 (Zhang et al. 2015). PEX5 ubiquitinated at K209 is recognized by SQSTM1 and targets peroxisomes for pexophagy. However, it has not been tested if NBR1 is involved in this ROS-driven pexophagy.

In general, PEX5 recognizes only conserved variants of a tripeptide of the peroxisomal targeting signal 1 (PTS1) at the extreme C-terminus, but surprisingly PEX5 interacted with internal PTS1-like sequences in TSC2 and ATM (Zhang et al. 2013; Zhang et al. 2015). Targeting of Rheb to peroxisomes is still mysterious, since it does not contain predicted PTS sequences. Several pathogenic TSC2 mutations identified in the internal PTS1-like sequence reduced association with PEX5 and peroxisomal localization of TSC2. Therefore, these mutations abrogated the ability of TSC 2 to suppress mTORC 1 by ROS. Mutation of the PTS1 affected ATM recruitment to peroxisomes but not its activation by ROS. In general, mTORC1 repression by ROS was reduced in peroxisome-deficient cells. However, it remains an open question why and how PEX5 as peroxisomal matrix protein import receptor delivers TSC2, Rheb, and ATM to the cytosolic face of peroxisomes but not into their matrix. 

generated by various peroxisomal oxidases (Fransen et al. 2012), has recently been shown to be involved in pexophagy during serum starvation in HepG2 and RPE-1 cells (Lee et al. 2018). Catalase knockdown by siRNAs or pharmacological inhibition by 3-amino-1-2-4-triazole (3AT) decreased peroxisome number in serum-depleted but not in serum-containing medium. Peroxisome abundance was assessed by immunoblotting and immunofluorescence using antibodies against the peroxisomal membrane proteins ATP-binding cassette subfamily D member 3 (ABCD3) and PEX14. Increased pexophagy upon catalase knockdown or inhibition was measured using the tandem fluorochrome pexophagy assay with mRFPEGFP-SKL as reporter. In this assay the "red-green" peroxisomal reporter becomes "red" only after the delivery of the peroxisome to the lysosome, whose $\mathrm{pH}$ is acidic and causes quenching of the EGFP fluorescence (Nazarko et al. 2014). In addition, catalase inhibition in the absence of serum increased the colocalization of NBR1 and ABCD3 as well as the protein levels of membrane-bound PEX5, whereas cytosolic PEX5 levels were decreased in the presence of the autophagy inhibitor chloroquine. Catalase inhibition increased ROS accumulation in peroxisomes, and the antioxidant N-acetyl-L-cysteine prevented this ROS increase and catalase inhibition-induced pexophagy (Lee et al. 2018). The detailed mechanisms how catalase inhibition and concomitant ROS accumulation mediate pexophagy need to be elucidated. However, since PEX5 ubiquitination in cells treated with 3AT was observed, it should be determined which site of PEX5 is ubiquitinated and if ubiquitination is mediated through ATM kinase activation. Nevertheless, why catalase inhibition-induced pexophagy was only observed under serum starvation remains enigmatic.

It is unknown whether other peroxisomal proteins besides PEX5 are ubiquitinated to signal pexophagy. In addition, the E3 ubiquitin ligase mediating PEX5 ubiquitination for pexophagy remained elusive. The peroxisomal membrane harbors three RING-finger E3 ubiquitin ligases, namely PEX2, PEX10, and PEX12, that form a transmembrane complex that has active sites located at the outer surface of the peroxisomal membrane (El Magraoui et al. 2012). These E3 ligases function primarily in the PEX5 matrix protein import cycle (Platta et al. 2009; Wang and Subramani 2017). Sargent et al. (Sargent et al. 
2016) showed that overexpression of PEX2, but not PEX10 or PEX12, triggers NBR1-dependent pexophagy (Fig. 2b). In addition, PEX2 is required for basal and amino acid starvation-induced pexophagy (Sargent et al. 2016). PEX5 and ABCD3 were ubiquitinated by PEX2 during amino acid starvation, but data suggest that also other peroxisomal membrane proteins might be ubiquitinated by PEX2. Moreover, the site of PEX5 ubiquitination for amino acid starvation-induced pexophagy needs to be identified. PEX2 is expressed at low levels under normal growth conditions, probably to prevent unintended pexophagy, but the levels are sufficient to form the E3 complex with PEX10 and PEX12 for PEX5 recycling. However, PEX2 expression was biphasic during amino acid starvation or mTORC1 inhibition. PEX2 protein levels rapidly increased during the first 2 hours of amino acid starvation before returning to the basal level (Sargent et al. 2016). The mechanism by which mTORC1 regulates PEX2 expression is unclear, but it has been hypothesized that mTORC1 accelerates PEX2 degradation by the proteasome.

Recently, a study showed that PEX5 depletion suppresses serum starvation-induced autophagy by downregulating the mTORC1 inhibitor TSC2 (Eun et al. 2018). The transcription factor EB (TFEB), a master regulator of lysosomal biogenesis and autophagy, is phosphorylated by mTORC1 and retained in the cytoplasm under normal growth conditions, whereas dephosphorylated TFEB translocates to the nucleus to induce the transcription of target genes under starvation (Napolitano and Ballabio 2016). TFEB nuclear translocation in response to serum starvation was suppressed by PEX5 knockdown, leading to impaired lysosomal biogenesis and function (Eun et al. 2018). Surprisingly, both PEX5 and TFEB depletion decreased the mRNA levels of several peroxisomal genes as well as positive regulators of peroxisome biogenesis, namely peroxisome proliferator-activated receptor alpha (PPAR $\alpha)$ and PPAR gamma coactivator 1-alpha $(P G C-1 \alpha)$, during starvation. Inhibition of mTORC1 restored TFEB localization and the function of peroxisomes and lysosomes in PEX5-depleted cells during serum starvation. Hence, induction of autophagy by mTORC1 inhibition could be a therapeutic option for Zellweger syndrome patients with PEX5 mutations (e.g., to prevent accumulation of abnormal mitochondria). 
The majority of peroxisomal biogenesis disorders (PBDs) results from mutations in one of 3 genes that encode the AAA ATPase complex (i.e., PEX1, PEX6, and PEX26) required for recycling of PEX5 into the cytosol after peroxisomal matrix protein import (Grimm et al. 2016; Steinberg et al. 2006). Recently, it has been shown that loss of the AAA-complex does not prevent matrix protein import, but instead leads to the accumulation of ubiquitinated PEX5 in the peroxisomal membrane that triggers NBR1-dependent pexophagy (Law et al. 2017) (Fig. 2b). Strikingly, inhibition of autophagy restored peroxisome number, protein import and function in fibroblasts of a patient with the most common PBD mutation, $P E X 1^{G 843 D}$. It has been proposed that the AAA-complex is a peroxisomal quality control factor that prevents pexophagy by removing ubiquitinated PEX5 from peroxisomes. It is unknown whether other ubiquitinated peroxisomal proteins could also be removed by the AAA-complex. The recently developed murine model of human PEX1 ${ }^{G 843 D}$ PBD, the PEX1 $1^{G 844 D}$ mouse (Hiebler et al. 2014), will be important to examine whether autophagy inhibition might be a therapeutic strategy for treating mild forms of PBDs caused by AAAcomplex dysfunction.

Recently, analysis of the protein interaction network of the poly(ADP-ribose) polymerase (PARP) family members tankyrase 1 (TNKS) and tankyrase 2 (TNKS2) revealed their association with PEX14 and localization on peroxisomes ( $\mathrm{Li}$ et al. 2017) (Fig. 2b). PARPs use nicotinamide adenine dinucleotide to modify substrate proteins with ADP-ribose modifications, and TNKS-mediated PARylation of substrates leads to two possible outcomes: a change in their localization or proteasome-dependent degradation. TNKS/TNKS2 enzyme activity was not required for its association with PEX14, and deletion of two TNKSbinding motifs in PEX14 disrupted its association with TNKS/TNKS2 and their localization on peroxisomes. Overexpression of TNKS/TNKS2 decreased the number of peroxisomes in cells via pexophagy and led to an enlargement of peroxisomes. However, the enzyme activities of TNKS/TNKS2 were not required for pexophagy. TNKS/TNKS2 are also involved in amino acid starvation-induced pexophagy, since loss of TNKS/TNKS2 prevented pexophagy under this condition. Since TNKS/TNKS2 are not ubiquitin-binding proteins and no interaction between TNKS/TNKS2 and the autophagy receptors NBR1 and SQSTM1 could be detected, TNKS/TNKS2-mediated pexophagy can be regarded as a non- 
canonical process. It has been suggested that an ATG9A-TNKS/TNKS2-PEX14 complex may serve as a receptor to induce pexophagy, where TNKS and TNKS2 promote pexophagy by associating with PEX14 on the peroxisome and recruiting the autophagic machinery through their association with ATG9A (Li et al. 2017) (Fig. 2b). However, the observed association of TNKS/TNKS2 with ATG9A raises the question how this interaction connects peroxisomes to the core autophagic machinery. ATG9 is the only multipass transmembrane ATG protein and is found in the Golgi complex under normal conditions. During autophagy, ATG9 vesicles shuttle around the forming phagophores and interact transiently, but ATG9 does not become a stable component of the autophagosome membrane (Mercer et al. 2018). It has been suggested that ATG9 delivers membrane to the forming phagophore and autophagosome. Emerging evidence has revealed the pathological relevance of TNKS/TNKS2 and identified these enzymes as potential drug targets. It would be interesting to examine whether TNKS/TNKS2-mediated pexophagy plays a role in the etiology of diseases such as neurodegenerative and developmental disorders or cancer.

The deubiquitylase ubiquitin carboxyl-terminal hydrolase 30 (USP30) has previously been shown to be constitutively associated with the outer mitochondrial membrane (OMM) and to counteract PTENinduced putative kinase 1 (PINK1) and parkin-dependent mitophagy by deubiquitylating OMM proteins (Bingol et al. 2014). Mitochondrial damage stimulates the E3 ubiquitin ligase parkin to assemble Lys6, Lys11, and Lys63 chains on mitochondria, and USP30 has a strong preference for cleaving Lys6- and Lys11-linked multimers and thereby inhibiting parkin-mediated mitophagy (Cunningham et al. 2015). In addition, substituting the mitochondrial targeting sequence of USP30 for a peroxisomal targeting sequence limits basal pexophagy, indicating that deubiquitylating surveillance by USP30 may be a general homeostatic mechanism to limit organellar autophagy (Cunningham et al. 2015). A recent study showed that USP30 regulates basal mitophagy in a PINK1-dependent but Parkin-independent manner, suggesting that USP30 could determine the threshold for mitophagy initiation by suppressing basal ubiquitination of specific OMM proteins (Marcassa et al. 2018). Interestingly, the authors identified a small fraction of endogenous USP30 that is targeted to peroxisomes independently of mitochondria (Marcassa et al. 2018). Neither the VPS35-dependent nor the VPS35-independent mitochondrial-derived vesicle pathways 
(Braschi et al. 2010; Neuspiel et al. 2008; Sugiura et al. 2017), which have been described to transport certain PEX proteins or the mitochondrial-anchored protein ligase (MAPL, also called Mul1 or Mulan) from mitochondria to peroxisomes, are involved in the transport of USP30 to peroxisomes. Peroxisomal USP30 displays properties of an integral membrane protein and protease protection assays indicate that its catalytic domain is exposed to the cytosol. USP30 localization to mitochondria and peroxisomes relies on distinct targeting sequences, however, the $\mathrm{N}$-terminal region encompassing amino acids 1-53 including a transmembrane domain is both necessary and sufficient for targeting USP30 to peroxisomes (Marcassa et al. 2018). It needs to be determined if USP30 is targeted to peroxisomes via PEX3 and PEX19, like most PMPs. Interestingly, USP30 depletion with siRNAs or USP30 knockout using CRISPR/Cas9 resulted in an approximately two-fold increase in basal mitophagy and pexophagy. However, peroxisome abundance remained unchanged, probably because the increased turnover of peroxisomes in USP3O knockdown and knockout cells is balanced by a corresponding increased rate in biogenesis. In addition, the fact that only catalytically active USP30 can restore pexophagy to baseline levels suggests that basal pexophagy is regulated by ubiquitination. However, peroxisomal membrane proteins that are targets of USP30 as well as the E3 ubiquitin ligase USP30 is opposing remain to be elucidated.

Peroxisomal function depends highly on molecular oxygen $\left(\mathrm{O}_{2}\right)$ due to its oxidative type of metabolism (Schönenberger and Kovacs 2015). Central to the molecular mechanisms underlying $\mathrm{O}_{2}$ homeostasis are the hypoxia-inducible factors-1 and -2 alpha (HIF-1 $\alpha$ and EPAS1/HIF-2 $\alpha$ ) that function as master regulators of the adaptive response to hypoxia. The HIF- $\alpha$ subunits are targeted by the von Hippel-Lindau (VHL) protein for ubiquitination and subsequent proteasomal degradation under normoxia. Hypoxia or loss of VHL function results in the stabilization of the HIF- $\alpha$ subunits and activation of their signaling. While it has been known for some time that HIF-1 $\alpha$ triggers autophagic clearance of mitochondria by activating the selective autophagy receptors BNIP3 (Bcl-2 and adenovirus E1B 19-kDainteracting protein 3), BNIP3-like (BNIP3L/NIX), and FUNDC1 (FUN14 domain containing 1), only recently a study linked HIF signaling to peroxisomes (Schönenberger and Kovacs 2015; Walter et al. 2014). 

control and liver-specific $\mathrm{Vhl}^{--}, \mathrm{Vhl}^{-/} / \mathrm{Hifla}^{-/}$, and $\mathrm{Vhl}^{-/} / \mathrm{Epas}^{-/}$mice. Peroxisome abundance was significantly decreased in livers of $\mathrm{Vhl}^{-/}$and $\mathrm{Vhl}^{-/} / \mathrm{Hifla}^{-/-}$mice by HIF-2 $\alpha$-mediated pexophagy, whereas peroxisome abundance was similar in control and $\mathrm{Vhl}^{-/} /$Epas $^{-/-}$mice (Fig. 2b). Peroxisome abundance and protein levels of NBR1 and SQSTM1 were concomitantly decreased in $V h l^{--}$and $V h l^{\digamma^{-} / H i f l a a^{--}}$mice. NBR1 and SQSTM1 colocalized with peroxisomes in $V h l^{-/}$livers, but surprisingly NBR1 already localized to peroxisomes in control livers (Walter et al. 2014). NBR1 might bind to an yet unidentified ubiquitinated PMP or is recruited to peroxisomes independently of ubiquitin via its amphipathic $\alpha$-helical membraneinteracting domain (J domain), capable of binding to the peroxisomal lipid bilayer (Deosaran et al. 2013). Neither peroxisome abundance nor NBR1 and SQSTM1 levels declined in $V h l^{-/}$mice treated with the autophagy inhibitor 3-methyladenine (3-MA), showing that the abundance of these autophagy receptors and peroxisomes are interconnected. However, 3-MA treatment led to a significant clustering of NBR1and SQSTM1-positive peroxisomes, suggesting that accumulation of NBR1 and SQSTM1 at peroxisomes and multimerization of these receptors might induce peroxisome clustering and prime peroxisomes for pexophagy (Fig. 2b).

It remains an open question how HIF-2 $\alpha$ induces pexophagy, but three models have been proposed (Schönenberger and Kovacs 2015; Schönenberger et al. 2015). First, HIF-2 $\alpha$ might induce an E3 ubiquitin ligase that ubiquitinates a PMP that enhances the recruitment of NBR1 to the peroxisome surface. Accumulation of NBR1 on peroxisomes likely recruits SQSTM1 and subsequently leads to clustering of peroxisomes and pexophagy. Second, NBR1 could be recruited to peroxisomes independently of ubiquitin via its $\mathbf{J}$ domain. HIF-2 $\alpha$ might induce or inhibit a kinase/phosphatase that leads to a change in the posttranslational modification of peroxisome-bound NBR1 and thereby triggers recruitment of the autophagic machinery. Third, HIF-2 $\alpha$-mediated pexophagy might be a 2 -step process where HIF-2 $\alpha$ induces both an E3 ubiquitin ligase to increase ubiquitination of $\operatorname{PMP}(\mathrm{s})$ and activates or inhibits a 
kinase/phosphatase to cause posttranslational modifications of autophagy receptors, PMPs, or ubiquitin that finally results in pexophagy.

Loss of VHL function occurs in up to $90 \%$ of sporadic human clear cell renal cell carcinomas (ccRCC), and HIF-2 $\alpha$ is considered to be a driver oncoprotein for ccRCC. Peroxisome abundance is reduced in VHL-deficient human ccRCC characterized by high HIF-2 $\alpha$ levels (Walter et al. 2014). However, whether reduction in peroxisome abundance represents a critical aspect of ccRCC progression remains to be explored. Since HIF-2 $\alpha$ stabilization is observed in the vast majority of solid tumors and a decrease in peroxisome abundance has been observed in various tumor cells, it has been proposed that HIF$2 \alpha$-mediated pexophagy might also influence tumor progression in other cancer types (Schönenberger and Kovacs 2015).

Two studies identified a peroxisome-ER contact site in human cells held together by a tethering complex of the peroxisomal membrane protein acyl-CoA binding domain 5 (ACBD5) and the ER resident vesicle-associated membrane protein-associated proteins A/B (VAPA/B) (Costello et al. 2017; Hua et al. 2017). ACBD5, the human Atg37 orthologue, has been reported as an essential pexophagy factor in yeast and mammalian cells in one study (Nazarko et al. 2014). However, two studies showed that ACBD5 deficiency impairs peroxisomal $\beta$-oxidation of very long-chain fatty acids, but it does not affect pexophagy (Ferdinandusse et al. 2017; Yagita et al. 2017). Mutations in ACBD5 and VAPB are linked to neuropathological disorders, and overexpression of an amyotrophic lateral sclerosis-associated VAPB mutant led to clustering of peroxisomes in an ACBD5-dependent manner (Hua et al. 2017). Since peroxisome clustering precedes degradation via pexophagy (Deosaran et al. 2013; Sargent et al. 2016) further studies are required to decipher the role of ACBD5 in mammalian pexophagy.

\section{Perspectives}

So far, only NBR1 and SQSTM1 have been described to act as selective autophagy receptors in mammalian pexophagy and it remains to be elucidated whether other receptors are involved in pexophagy. 

optineurin (OPTN), and Tax1-binding protein 1 (TAX1BP1), act as ubiquitin-binding proteins that mediate the interaction between ubiquitinated proteins and the core autophagy machinery. However, their role in pexophagy has not been examined so far. In general, it is important to decipher the level of redundancy of autophagic cargo receptors for several selective autophagy processes. Ubiquitination of PEX5 and ABCD3 in response to various stress conditions leads to mammalian pexophagy. However, other peroxisomal membrane proteins may also be ubiquitinated to initiate pexophagy. Thus, it will be important to identify ubiquitinated peroxisomal membrane proteins and their ubiquitination sites during pexophagy. In addition, it needs to be examined whether ubiquitination is the general degradative signal or whether the peroxisomal fate depends on the protein that is posttranslationally modified. However, it should be considered that several ubiquitin-independent selective autophagy pathways have been identified, such as ER-phagy and HIF-1 $\alpha$-mediated mitophagy (Khaminets et al. 2016; Khaminets et al. 2015; Mancias and Kimmelman 2016; Schönenberger and Kovacs 2015). For example, a study showed that externalization of cardiolipin to the outer mitochondrial membrane serves as signal for mitophagy (Chu et al. 2013). Hence, we cannot exclude the possibility that future studies discover ubiquitin-independent pexophagy pathways and receptors. Several stress conditions have been shown to stimulate pexophagy in yeast or mammalian cells, including hypoxia, oxidative stress, and nutrient deprivation. An important focus for future studies will be to clarify why these general perturbations of homeostasis cause pexophagy in some cell types, while nonspecific bulk autophagy or selective autophagy of other organelles (i.e., mitochondria) is triggered in others. It will be interesting to compare differences in the mechanisms that regulate clearance of different organelles, such as peroxisomes and mitochondria, in response to general stress conditions. Another major challenge is to provide evidence that the up to now described regulatory mechanisms of mammalian pexophagy are relevant in vivo.

It is not known if peroxisomal fission plays a role in mammalian pexophagy. DRP1/DLP1mediated fission facilitates mitophagy in mammalian cells by dividing mitochondria into fragments amenable to autophagosome engulfment and/or segregating damaged mitochondrial subdomains for 
eliminiation (Burman et al. 2017; Gomes and Scorrano 2013). Since fission is important for the progression of mitophagy in yeasts and mammals as well as pexophagy in yeasts, future studies should reveal if peroxisomal fission also plays a role in mammalian pexophagy.

\title{
Acknowledgements
}

This work was supported by the Swiss National Science Foundation (SNSF) grant 31003A_166245 to W.J.K.

\begin{abstract}
Abbreviations
3AT, 3-amino-1-2-4-triazole; 3-MA, 3-methyladenine; A11BR, Atg11-binding region; ABCD3, ATPbinding cassette subfamily D member 3; ACBD5, acyl-CoA binding protein 5; AIM, Atg8-interacting motif; ALOX15, 15-lipoxygenase-1; AMPK, AMP-activated protein kinase; ATG, autophagy-related gene; ATM, ataxia-telangiectasia mutated; BNIP3, Bcl-2 and adenovirus E1B 19-kDa-interacting protein 3; Caf4, CCR4-associated factor 4; CALCOCO2, calcium-binding and coiled-coil domain-containing protein 2; ccRCC, clear cell renal cell carcinoma; CHO, Chinese hamster ovary; Dnm1, dynamin 1; ER, endoplasmic reticulum; FUNDC1, FUN14 domain containing 1; GABARAP, $\gamma$-aminobutyric acid receptor-associated protein; HIF, hypoxia-inducible factor; Hrr25, casein kinase $1 \delta$ homologue; LC3, microtubule-associated protein-1 light chain 3; LIR, LC3-interacting regions; LKB1, serine/threonine kinase 11; MAPL, mitochondrial-anchored protein ligase; Mdv1, mitochondrial division protein 1; MIPA, micropexophagyspecific apparatus; mTORC1, mechanistic target of rapamycin complex 1; Mul1, mitochondrial-anchored protein ligase; Mulan, mitochondrial-anchored protein ligase; NBR1, neighbor of BRCA1 gene; NDP52, nuclear dot protein $52 \mathrm{kDa}$; OMM, outer mitochondrial membrane; OPTN, optineurin; p62, sequestosome 1; PARP, poly(ADP-ribose) polymerase; PAS, phagophore assembly site; PBD, peroxisome biogenesis disorder; PE, phosphatidylethanolamine; PEX, peroxin; PGC-1 $\alpha$, PPAR gamma coactivator 1-alpha; PI3K, phosphatidylinositol 3-kinase; PIK3R4, PI3K regulatory subunit 4; PINK1, PTEN-induced putative kinase
\end{abstract}


1; PMP, peroxisomal membrane protein; PPAR $\alpha$, peroxisome proliferator-activated receptor alpha; PTS1, peroxisomal targeting signal 1; RHEB, RAS homolog enriched in brain; ROS, reactive oxygen species; RPC, receptor protein complex; SQSTM1, sequestosome 1; TAX1BP1, Tax1-binding protein 1; TFEB, transcription factor EB; TNKS, tankyrase; TSC, tuberous sclerosis complex; Ub, ubiquitin; UBD, Ubbinding domain; ULK1/2, UNC-51-like kinase 1/2; USP30, ubiquitin carboxyl-terminal hydrolase 30; VAPA/B, vesicle-associated membrane protein-associated proteins A/B; VHL, von Hippel-Lindau; Vps, vacuolar protein sorting-associated protein. 


\section{Figure legend}

Figure 1. Mechanisms of macroautophagy (a) and selective autophagy (b). (a) Schematic representation of macroautophagy (autophagy). The autophagy process can be divided into at least six sequential steps: (1) initiation, (2) membrane nucleation and phagophore formation, (3) phagophore elongation with concomitant cargo sequestration, (4) phagophore closure forming an autophagosome, (5) fusion of the autophagosome with the lysosome forming an autolysosome, and (6) degradation of sequestered cargo in the autolysosome. For a detailed description see main text. (b) Principles of Ub-dependent and Ubindependent selective autophagy. In Ub-dependent selective autophagy (left part), a prototypical selective autophagy receptor with an UBD recognizes Ub chains attached to intracellular cargo and physically links the targeted material to the autophagosomal membrane via a LIR motif that binds to lipidated and autophagosome membrane-associated LC3/GABARAP. In Ub-independent selective autophagy (right part), autophagy receptors (orange) directly bind to intracellular cargo (e.g., peroxisomes, mitochondria) and connect it to the autophagosomal membrane through their LIR motif. Autophagy receptors tend to cluster their cargo through specialized oligomerization domains and they are degraded together with their cargo within autolysosomes.

Figure 2. Mechanisms of pexophagy in yeast (a) and mammalian cells (b). For a detailed description see main text. (a) In yeast pexophagy can be triggered by a shift in nutrient conditions. Atg30 and Atg36 act as autophagy receptors in $P$. pastoris and S. cerevisiae, respectively, linking peroxisomes to the phagophore by binding at least one peroxin and Atg8. Atg11 functions as a scaffold for both P. pastoris and S. cerevisiae. Atg30 and Atg36 are phosphorylated by the kinase Hrr 25 to allow recruitment of the autophagic scaffold protein Atg11. In H. polymorpha, Pex3 is removed from the peroxisomal surface by the ubiquitinproteasome system prior to sequestration and degradation of the organelle by pexophagy. Pex14 is required for recognition of the peroxisome by the autophagic machinery, but a receptor protein (AtgX) to link the peroxisome to the phagophore has not been identified. (b) Several stress conditions have been shown to stimulate pexophagy in mammalian cells, including hypoxia, oxidative stress, nutrient deprivation, and 
peroxisomal dysfunctions (AAA-complex defects). So far, only the ubiquitin-binding autophagy receptors NBR1 and SQSTM1 have been described to tether peroxisomes to the phagophore. Ubiquitination of PEX5 and $\mathrm{ABCD} 3$ in response to various stress conditions leads to mammalian pexophagy, however, other peroxisomal membrane proteins may also be ubiquitinated to initiate pexophagy. PEX14 has also been reported to link peroxisomes to the phagophore either by directly binding LC3 family members or TNKS/TNKS2. To date, except for HIF-2 $\alpha$-induced pexophagy the mechanisms of mammalian pexophagy in response to other stressors have only been elucidated in vitro. 


\section{References}

Alexander A, Cai SL, Kim J, Nanez A, Sahin M, MacLean KH, Inoki K, Guan KL, Shen J, Person MD, Kusewitt D, Mills GB, Kastan MB, Walker CL (2010) ATM signals to TSC2 in the cytoplasm to regulate mTORC1 in response to ROS. Proc Natl Acad Sci U S A 107:4153-4158 doi:10.1073/pnas.0913860107

Ano Y, Hattori T, Kato N, Sakai Y (2005) Intracellular ATP correlates with mode of pexophagy in Pichia pastoris. Biosci Biotechnol Biochem 69:1527-1533 doi:10.1271/bbb.69.1527

Bellu AR, Komori M, van der Klei IJ, Kiel JA, Veenhuis M (2001a) Peroxisome biogenesis and selective degradation converge at Pex14p. J Biol Chem 276:44570-44574 doi:10.1074/jbc.M107599200

Bellu AR, Kram AM, Kiel JA, Veenhuis M, van der Klei IJ (2001b) Glucose-induced and nitrogenstarvation-induced peroxisome degradation are distinct processes in Hansenula polymorpha that involve both common and unique genes. FEMS Yeast Res 1:23-31

Bellu AR, Salomons FA, Kiel JA, Veenhuis M, Van Der Klei IJ (2002) Removal of Pex3p is an important initial stage in selective peroxisome degradation in Hansenula polymorpha. J Biol Chem 277:42875-42880 doi:10.1074/jbc.M205437200

Bingol B, Tea JS, Phu L, Reichelt M, Bakalarski CE, Song Q, Foreman O, Kirkpatrick DS, Sheng M (2014) The mitochondrial deubiquitinase USP30 opposes parkin-mediated mitophagy. Nature 510:370-375 doi:10.1038/nature13418

Birgisdottir AB, Lamark T, Johansen T (2013) The LIR motif - crucial for selective autophagy. J Cell Sci 126:3237-3247 doi:10.1242/jcs.126128

Braschi E, Goyon V, Zunino R, Mohanty A, Xu L, McBride HM (2010) Vps35 mediates vesicle transport between the mitochondria and peroxisomes. Curr Biol 20:1310-1315 doi:10.1016/j.cub.2010.05.066

Burman JL, Pickles S, Wang C, Sekine S, Vargas JNS, Zhang Z, Youle AM, Nezich CL, Wu X, Hammer JA, Youle RJ (2017) Mitochondrial fission facilitates the selective mitophagy of protein aggregates. J Cell Biol 216:3231-3247 doi:10.1083/jcb.201612106

Burnett SF, Farre JC, Nazarko TY, Subramani S (2015) Peroxisomal Pex3 activates selective autophagy of peroxisomes via interaction with the pexophagy receptor Atg30. J Biol Chem 290:8623-8631 doi:10.1074/jbc.M114.619338

Chiang HL, Schekman R, Hamamoto S (1996) Selective uptake of cytosolic, peroxisomal, and plasma membrane proteins into the yeast lysosome for degradation. J Biol Chem 271:9934-9941

Chu CT, Ji J, Dagda RK, Jiang JF, Tyurina YY, Kapralov AA, Tyurin VA, Yanamala N, Shrivastava IH, Mohammadyani D, Wang KZQ, Zhu J, Klein-Seetharaman J, Balasubramanian K, Amoscato AA, Borisenko G, Huang Z, Gusdon AM, Cheikhi A, Steer EK, Wang R, Baty C, Watkins S, Bahar I, Bayir H, Kagan VE (2013) Cardiolipin externalization to the outer mitochondrial membrane acts as an elimination signal for mitophagy in neuronal cells. Nat Cell Biol 15:1197-1205 doi: $10.1038 / \mathrm{ncb} 2837$

Cipolla CM, Lodhi IJ (2017) Peroxisomal Dysfunction in Age-Related Diseases. Trends Endocrinol Metab 28:297-308 doi:10.1016/j.tem.2016.12.003

Costello JL, Castro IG, Hacker C, Schrader TA, Metz J, Zeuschner D, Azadi AS, Godinho LF, Costina V, Findeisen P, Manner A, Islinger M, Schrader M (2017) ACBD5 and VAPB mediate membrane associations between peroxisomes and the ER. J Cell Biol 216:331-342 doi:10.1083/jcb.201607055

Cunningham CN, Baughman JM, Phu L, Tea JS, Yu C, Coons M, Kirkpatrick DS, Bingol B, Corn JE (2015) USP30 and parkin homeostatically regulate atypical ubiquitin chains on mitochondria. Nat Cell Biol 17:160-169 doi:10.1038/ncb3097

Deosaran E, Larsen KB, Hua R, Sargent G, Wang Y, Kim S, Lamark T, Jauregui M, Law K, LippincottSchwartz J, Brech A, Johansen T, Kim PK (2013) NBR1 acts as an autophagy receptor for peroxisomes. J Cell Sci 126:939-952 doi:10.1242/jcs.114819 
Dixit E, Boulant S, Zhang Y, Lee AS, Odendall C, Shum B, Hacohen N, Chen ZJ, Whelan SP, Fransen M, Nibert ML, Superti-Furga G, Kagan JC (2010) Peroxisomes are signaling platforms for antiviral innate immunity. Cell 141:668-681 doi:10.1016/j.cell.2010.04.018

Dorninger F, Wiesinger C, Braverman NE, Forss-Petter S, Berger J (2015) Ether lipid deficiency does not cause neutropenia or leukopenia in mice and men. Cell Metab 21:650-651 doi:10.1016/j.cmet.2015.04.014

El Magraoui F, Baumer BE, Platta HW, Baumann JS, Girzalsky W, Erdmann R (2012) The RING-type ubiquitin ligases Pex2p, Pex10p and Pex12p form a heteromeric complex that displays enhanced activity in an ubiquitin conjugating enzyme-selective manner. FEBS J 279:2060-2070 doi:10.1111/j.1742-4658.2012.08591.x

Eun SY, Lee JN, Nam IK, Liu ZQ, So HS, Choe SK, Park R (2018) PEX5 regulates autophagy via the mTORC1-TFEB axis during starvation. Exp Mol Med 50:4 doi:10.1038/s12276-017-0007-8

Farre JC, Burkenroad A, Burnett SF, Subramani S (2013) Phosphorylation of mitophagy and pexophagy receptors coordinates their interaction with Atg8 and Atg11. EMBO Rep 14:441-449 doi:10.1038/embor.2013.40

Farre JC, Manjithaya R, Mathewson RD, Subramani S (2008) PpAtg30 tags peroxisomes for turnover by selective autophagy. Dev Cell 14:365-376 doi:10.1016/j.devcel.2007.12.011

Farre JC, Subramani S (2016) Mechanistic insights into selective autophagy pathways: lessons from yeast. Nat Rev Mol Cell Biol 17:537-552 doi:10.1038/nrm.2016.74

Faust PL, Kovacs WJ (2014) Cholesterol biosynthesis and ER stress in peroxisome deficiency. Biochimie 98:75-85 doi:10.1016/j.biochi.2013.10.019

Ferdinandusse S, Falkenberg KD, Koster J, Mooyer PA, Jones R, van Roermund CWT, Pizzino A, Schrader M, Wanders RJA, Vanderver A, Waterham HR (2017) ACBD5 deficiency causes a defect in peroxisomal very long-chain fatty acid metabolism. J Med Genet 54:330-337 doi:10.1136/jmedgenet-2016-104132

Fransen M, Lismont C, Walton P (2017) The Peroxisome-Mitochondria Connection: How and Why? Int J Mol Sci 18 doi:10.3390/ijms18061126

Fransen M, Nordgren M, Wang B, Apanasets O (2012) Role of peroxisomes in ROS/RNS-metabolism: implications for human disease. Biochim Biophys Acta 1822:1363-1373 doi:10.1016/j.bbadis.2011.12.001

Fransen M, Nordgren M, Wang B, Apanasets O, Van Veldhoven PP (2013) Aging, age-related diseases and peroxisomes. Subcell Biochem 69:45-65 doi:10.1007/978-94-007-6889-5_3

Galluzzi L, Baehrecke EH, Ballabio A, Boya P, Bravo-San Pedro JM, Cecconi F, Choi AM, Chu CT, Codogno P, Colombo MI, Cuervo AM, Debnath J, Deretic V, Dikic I, Eskelinen EL, Fimia GM, Fulda S, Gewirtz DA, Green DR, Hansen M, Harper JW, Jaattela M, Johansen T, Juhasz G, Kimmelman AC, Kraft C, Ktistakis NT, Kumar S, Levine B, Lopez-Otin C, Madeo F, Martens S, Martinez J, Melendez A, Mizushima N, Munz C, Murphy LO, Penninger JM, Piacentini M, Reggiori F, Rubinsztein DC, Ryan KM, Santambrogio L, Scorrano L, Simon AK, Simon HU, Simonsen A, Tavernarakis N, Tooze SA, Yoshimori T, Yuan J, Yue Z, Zhong Q, Kroemer G (2017) Molecular definitions of autophagy and related processes. EMBO J 36:1811-1836 doi:10.15252/embj.201796697

Gomes LC, Scorrano L (2013) Mitochondrial morphology in mitophagy and macroautophagy. Biochim Biophys Acta 1833:205-212 doi:10.1016/j.bbamcr.2012.02.012

Grimm I, Erdmann R, Girzalsky W (2016) Role of AAA(+)-proteins in peroxisome biogenesis and function. Biochim Biophys Acta 1863:828-837 doi:10.1016/j.bbamcr.2015.10.001

Hara-Kuge S, Fujiki Y (2008) The peroxin Pex14p is involved in LC3-dependent degradation of mammalian peroxisomes. Exp Cell Res 314:3531-3541 doi:10.1016/j.yexcr.2008.09.015

Hazra PP, Suriapranata I, Snyder WB, Subramani S (2002) Peroxisome remnants in pex3delta cells and the requirement of Pex $3 p$ for interactions between the peroxisomal docking and translocation subcomplexes. Traffic 3:560-574 
Hiebler S, Masuda T, Hacia JG, Moser AB, Faust PL, Liu A, Chowdhury N, Huang N, Lauer A, Bennett J, Watkins PA, Zack DJ, Braverman NE, Raymond GV, Steinberg SJ (2014) The Pex1-G844D mouse: a model for mild human Zellweger spectrum disorder. Mol Genet Metab 111:522-532 doi:10.1016/j.ymgme.2014.01.008

Hua R, Cheng D, Coyaud E, Freeman S, Di Pietro E, Wang Y, Vissa A, Yip CM, Fairn GD, Braverman N, Brumell JH, Trimble WS, Raught B, Kim PK (2017) VAPs and ACBD5 tether peroxisomes to the ER for peroxisome maintenance and lipid homeostasis. J Cell Biol 216:367-377 doi:10.1083/jcb.201608128

Hutchins MU, Veenhuis M, Klionsky DJ (1999) Peroxisome degradation in Saccharomyces cerevisiae is dependent on machinery of macroautophagy and the Cvt pathway. J Cell Sci 112 ( Pt 22):40794087

Huybrechts SJ, Van Veldhoven PP, Brees C, Mannaerts GP, Los GV, Fransen M (2009) Peroxisome dynamics in cultured mammalian cells. Traffic 10:1722-1733 doi:10.1111/j.16000854.2009.00970.x

Islinger M, Voelkl A, Fahimi HD, Schrader M (2018) The peroxisome: an update on mysteries 2.0. Histochem Cell Biol

Iwata J, Ezaki J, Komatsu M, Yokota S, Ueno T, Tanida I, Chiba T, Tanaka K, Kominami E (2006) Excess peroxisomes are degraded by autophagic machinery in mammals. J Biol Chem 281:40354041 doi:10.1074/jbc.M512283200

Jiang L, Hara-Kuge S, Yamashita S, Fujiki Y (2015) Peroxin Pex14p is the key component for coordinated autophagic degradation of mammalian peroxisomes by direct binding to LC3-II. Genes Cells 20:36-49 doi:10.1111/gtc.12198

Johansen T, Lamark T (2011) Selective autophagy mediated by autophagic adapter proteins. Autophagy 7:279-296

Khaminets A, Behl C, Dikic I (2016) Ubiquitin-Dependent And Independent Signals In Selective Autophagy. Trends Cell Biol 26:6-16 doi:10.1016/j.tcb.2015.08.010

Khaminets A, Heinrich T, Mari M, Grumati P, Huebner AK, Akutsu M, Liebmann L, Stolz A, Nietzsche S, Koch N, Mauthe M, Katona I, Qualmann B, Weis J, Reggiori F, Kurth I, Hubner CA, Dikic I (2015) Regulation of endoplasmic reticulum turnover by selective autophagy. Nature 522:354358 doi:10.1038/nature14498

Kiel JA, Emmrich K, Meyer HE, Kunau WH (2005) Ubiquitination of the peroxisomal targeting signal type 1 receptor, Pex 5p, suggests the presence of a quality control mechanism during peroxisomal matrix protein import. J Biol Chem 280:1921-1930 doi:10.1074/jbc.M403632200

Kim PK, Hailey DW, Mullen RT, Lippincott-Schwartz J (2008) Ubiquitin signals autophagic degradation of cytosolic proteins and peroxisomes. Proc Natl Acad Sci U S A 105:20567-20574 doi:10.1073/pnas.0810611105

Kirkin V, McEwan DG, Novak I, Dikic I (2009) A role for ubiquitin in selective autophagy. Mol Cell 34:259-269 doi:10.1016/j.molcel.2009.04.026

Klionsky DJ, Schulman BA (2014) Dynamic regulation of macroautophagy by distinctive ubiquitin-like proteins. Nat Struct Mol Biol 21:336-345 doi:10.1038/nsmb.2787

Law KB, Bronte-Tinkew D, Di Pietro E, Snowden A, Jones RO, Moser A, Brumell JH, Braverman N, Kim PK (2017) The peroxisomal AAA ATPase complex prevents pexophagy and development of peroxisome biogenesis disorders. Autophagy 13:868-884 doi:10.1080/15548627.2017.1291470

Leao AN, Kiel JA (2003) Peroxisome homeostasis in Hansenula polymorpha. FEMS Yeast Res 4:131139

Lee JN, Dutta RK, Maharjan Y, Liu ZQ, Lim JY, Kim SJ, Cho DH, So HS, Choe SK, Park R (2018) Catalase inhibition induces pexophagy through ROS accumulation. Biochem Biophys Res Commun 501:696-702 doi:10.1016/j.bbrc.2018.05.050

Li X, Han H, Zhou MT, Yang B, Ta AP, Li N, Chen J, Wang W (2017) Proteomic Analysis of the Human Tankyrase Protein Interaction Network Reveals Its Role in Pexophagy. Cell Rep 20:737-749 doi:10.1016/j.celrep.2017.06.077 
Lodhi IJ, Link DC, Semenkovich CF (2015a) Acute ether lipid deficiency affects neutrophil biology in mice. Cell Metab 21:652-653 doi:10.1016/j.cmet.2015.04.018

Lodhi IJ, Wei X, Yin L, Feng C, Adak S, Abou-Ezzi G, Hsu FF, Link DC, Semenkovich CF (2015b) Peroxisomal lipid synthesis regulates inflammation by sustaining neutrophil membrane phospholipid composition and viability. Cell Metab 21:51-64 doi:10.1016/j.cmet.2014.12.002

Mancias JD, Kimmelman AC (2016) Mechanisms of Selective Autophagy in Normal Physiology and Cancer. J Mol Biol 428:1659-1680 doi:10.1016/j.jmb.2016.02.027

Manivannan S, de Boer R, Veenhuis M, van der Klei IJ (2013) Lumenal peroxisomal protein aggregates are removed by concerted fission and autophagy events. Autophagy 9:1044-1056 doi:10.4161/auto.24543

Mao K, Liu X, Feng Y, Klionsky DJ (2014) The progression of peroxisomal degradation through autophagy requires peroxisomal division. Autophagy 10:652-661 doi:10.4161/auto.27852

Mao K, Wang K, Liu X, Klionsky DJ (2013) The scaffold protein Atg11 recruits fission machinery to drive selective mitochondria degradation by autophagy. Dev Cell 26:9-18 doi:10.1016/j.devcel.2013.05.024

Marcassa E, Kallinos A, Jardine J, Rusilowicz-Jones EV, Martinez A, Kuehl S, Islinger M, Clague MJ, Urbe S (2018) Dual role of USP30 in controlling basal pexophagy and mitophagy. EMBO Rep 19 doi:10.15252/embr.201745595

McEwan DG, Dikic I (2011) The Three Musketeers of Autophagy: phosphorylation, ubiquitylation and acetylation. Trends Cell Biol 21:195-201 doi:10.1016/j.tcb.2010.12.006

Mercer TJ, Gubas A, Tooze SA (2018) A molecular perspective of mammalian autophagosome biogenesis. J Biol Chem 293:5386-5395 doi:10.1074/jbc.R117.810366

Mizushima N, Yoshimori T, Ohsumi Y (2011) The role of Atg proteins in autophagosome formation. Annu Rev Cell Dev Biol 27:107-132 doi:10.1146/annurev-cellbio-092910-154005

Monastryska I, Sjollema K, van der Klei IJ, Kiel JA, Veenhuis M (2004) Microautophagy and macropexophagy may occur simultaneously in Hansenula polymorpha. FEBS Lett 568:135-138 doi:10.1016/j.febslet.2004.05.018

Monastyrska I, Klionsky DJ (2006) Autophagy in organelle homeostasis: peroxisome turnover. Mol Aspects Med 27:483-494 doi:10.1016/j.mam.2006.08.004

Motley AM, Nuttall JM, Hettema EH (2012a) Atg36: the Saccharomyces cerevisiae receptor for pexophagy. Autophagy 8:1680-1681 doi:10.4161/auto.21485

Motley AM, Nuttall JM, Hettema EH (2012b) Pex3-anchored Atg36 tags peroxisomes for degradation in Saccharomyces cerevisiae. EMBO J 31:2852-2868 doi:10.1038/emboj.2012.151

Napolitano G, Ballabio A (2016) TFEB at a glance J Cell Sci 129:2475-2481 doi:10.1242/jcs.146365

Nazarko TY (2014) Atg37 regulates the assembly of the pexophagic receptor protein complex. Autophagy 10:1348-1349 doi:10.4161/auto.29073

Nazarko TY, Ozeki K, Till A, Ramakrishnan G, Lotfi P, Yan M, Subramani S (2014) Peroxisomal Atg37 binds Atg30 or palmitoyl-CoA to regulate phagophore formation during pexophagy. J Cell Biol 204:541-557 doi:10.1083/jcb.201307050

Neuspiel M, Schauss AC, Braschi E, Zunino R, Rippstein P, Rachubinski RA, Andrade-Navarro MA, McBride HM (2008) Cargo-selected transport from the mitochondria to peroxisomes is mediated by vesicular carriers. Curr Biol 18:102-108 doi:10.1016/j.cub.2007.12.038

Noda NN, Inagaki F (2015) Mechanisms of Autophagy. Annu Rev Biophys 44:101-122 doi:10.1146/annurev-biophys-060414-034248

Nordgren M, Francisco T, Lismont C, Hennebel L, Brees C, Wang B, Van Veldhoven PP, Azevedo JE, Fransen M (2015) Export-deficient monoubiquitinated PEX5 triggers peroxisome removal in SV40 large T antigen-transformed mouse embryonic fibroblasts. Autophagy 11:1326-1340 doi:10.1080/15548627.2015.1061846

Nordgren M, Fransen M (2014) Peroxisomal metabolism and oxidative stress. Biochimie 98:56-62 doi:10.1016/j.biochi.2013.07.026 
Nuttall JM, Motley AM, Hettema EH (2014) Deficiency of the exportomer components Pex1, Pex6, and Pex15 causes enhanced pexophagy in Saccharomyces cerevisiae. Autophagy 10:835-845 doi:10.4161/auto. 28259

Odendall C, Dixit E, Stavru F, Bierne H, Franz KM, Durbin AF, Boulant S, Gehrke L, Cossart P, Kagan JC (2014) Diverse intracellular pathogens activate type III interferon expression from peroxisomes. Nat Immunol 15:717-726 doi:10.1038/ni.2915

Oku M, Sakai Y (2016) Pexophagy in yeasts. Biochim Biophys Acta 1863:992-998 doi:10.1016/j.bbamcr.2015.09.023

Platta HW, El Magraoui F, Baumer BE, Schlee D, Girzalsky W, Erdmann R (2009) Pex2 and pex12 function as protein-ubiquitin ligases in peroxisomal protein import. Mol Cell Biol 29:5505-5516 doi:10.1128/MCB.00388-09

Poole B, Leighton F, De Duve C (1969) The synthesis and turnover of rat liver peroxisomes. II. Turnover of peroxisome proteins. J Cell Biol 41:536-546

Puri P, Baillie RA, Wiest MM, Mirshahi F, Choudhury J, Cheung O, Sargeant C, Contos MJ, Sanyal AJ (2007) A lipidomic analysis of nonalcoholic fatty liver disease. Hepatology 46:1081-1090 doi:10.1002/hep. 21763

Puri P, Wiest MM, Cheung O, Mirshahi F, Sargeant C, Min HK, Contos MJ, Sterling RK, Fuchs M, Zhou H, Watkins SM, Sanyal AJ (2009) The plasma lipidomic signature of nonalcoholic steatohepatitis. Hepatology 50:1827-1838 doi:10.1002/hep.23229

Raymond GV, Watkins P, Steinberg S, Powers J (2009) Peroxisomal disorders. In: Lajtha A, Tettamanti G, Goracci G (eds) Handbook of Neurochemistry and Molecular Neurobiology: Neural Lipids. Springer Science+Business Media, Berlin, pp 631-670. doi:10.1007/978-0-387-30378-9_26

Sargent G, van Zutphen T, Shatseva T, Zhang L, Di Giovanni V, Bandsma R, Kim PK (2016) PEX2 is the E3 ubiquitin ligase required for pexophagy during starvation. J Cell Biol 214:677-690 doi:10.1083/jcb.201511034

Schönenberger MJ, Kovacs WJ (2015) Hypoxia signaling pathways: modulators of oxygen-related organelles. Front Cell Dev Biol 3:42 doi:10.3389/fcell.2015.00042

Schönenberger MJ, Krek W, Kovacs WJ (2015) EPAS1/HIF-2alpha is a driver of mammalian pexophagy. Autophagy 11:967-969 doi:10.1080/15548627.2015.1045180

Schrader M, Bonekamp NA, Islinger M (2012) Fission and proliferation of peroxisomes. Biochim Biophys Acta 1822:1343-1357 doi:10.1016/j.bbadis.2011.12.014

Schrader M, Fahimi HD (2006) Peroxisomes and oxidative stress. Biochim Biophys Acta 1763:17551766 doi:10.1016/j.bbamcr.2006.09.006

Schrader M, Fahimi HD (2008) The peroxisome: still a mysterious organelle. Histochem Cell Biol 129:421-440 doi:10.1007/s00418-008-0396-9

Schreiber A, Peter M (2014) Substrate recognition in selective autophagy and the ubiquitin-proteasome system. Biochim Biophys Acta 1843:163-181 doi:10.1016/j.bbamcr.2013.03.019

Shaid S, Brandts CH, Serve H, Dikic I (2013) Ubiquitination and selective autophagy. Cell Death Differ 20:21-30 doi:10.1038/cdd.2012.72

Steinberg SJ, Dodt G, Raymond GV, Braverman NE, Moser AB, Moser HW (2006) Peroxisome biogenesis disorders. Biochim Biophys Acta 1763:1733-1748 doi:10.1016/j.bbamcr.2006.09.010

Sugiura A, Mattie S, Prudent J, McBride HM (2017) Newly born peroxisomes are a hybrid of mitochondrial and ER-derived pre-peroxisomes. Nature 542:251-254 doi:10.1038/nature21375

Tanaka C, Tan LJ, Mochida K, Kirisako H, Koizumi M, Asai E, Sakoh-Nakatogawa M, Ohsumi Y, Nakatogawa H (2014) Hrr25 triggers selective autophagy-related pathways by phosphorylating receptor proteins. J Cell Biol 207:91-105 doi:10.1083/jcb.201402128

Tuttle DL, Dunn WA, Jr. (1995) Divergent modes of autophagy in the methylotrophic yeast Pichia pastoris. J Cell Sci 108 ( Pt 1):25-35

Van Veldhoven PP (2010) Biochemistry and genetics of inherited disorders of peroxisomal fatty acid metabolism. J Lipid Res 51:2863-2895 doi:10.1194/jlr.R005959 
van Zutphen T, van der Klei IJ, Kiel JA (2008) Pexophagy in Hansenula polymorpha. Methods Enzymol 451:197-215 doi:10.1016/S0076-6879(08)03214-X

van Zutphen T, Veenhuis M, van der Klei IJ (2011) Damaged peroxisomes are subject to rapid autophagic degradation in the yeast Hansenula polymorpha. Autophagy 7:863-872

Veenhuis M, Komori M, Salomons F, Hilbrands RE, Hut H, Baerends RJ, Kiel JA, van der Klei IJ (1996) Peroxisomal remnants in peroxisome-deficient mutants of the yeast Hansenula polymorpha [corrected]. FEBS Lett 383:114-118

Walker CL, Pomatto LCD, Tripathi DN, Davies KJA (2018) Redox Regulation of Homeostasis and Proteostasis in Peroxisomes. Physiol Rev 98:89-115 doi:10.1152/physrev.00033.2016

Walter KM, Schönenberger MJ, Trotzmuller M, Horn M, Elsasser HP, Moser AB, Lucas MS, Schwarz T, Gerber PA, Faust PL, Moch H, Kofeler HC, Krek W, Kovacs WJ (2014) Hif-2alpha promotes degradation of mammalian peroxisomes by selective autophagy. Cell Metab 20:882-897 doi:10.1016/j.cmet.2014.09.017

Wang W, Subramani S (2017) Role of PEX5 ubiquitination in maintaining peroxisome dynamics and homeostasis. Cell Cycle 16:2037-2045 doi:10.1080/15384101.2017.1376149

Williams C, van der Klei IJ (2013) Pexophagy-linked degradation of the peroxisomal membrane protein Pex3p involves the ubiquitin-proteasome system. Biochem Biophys Res Commun 438:395-401 doi:10.1016/j.bbrc.2013.07.086

Yagita Y, Shinohara K, Abe Y, Nakagawa K, Al-Owain M, Alkuraya FS, Fujiki Y (2017) Deficiency of a Retinal Dystrophy Protein, Acyl-CoA Binding Domain-containing 5 (ACBD5), Impairs Peroxisomal beta-Oxidation of Very-long-chain Fatty Acids. J Biol Chem 292:691-705 doi:10.1074/jbc.M116.760090

Yamashita S, Abe K, Tatemichi Y, Fujiki Y (2014) The membrane peroxin PEX3 induces peroxisomeubiquitination-linked pexophagy. Autophagy 10:1549-1564 doi:10.4161/auto.29329

Yokota S, Dariush Fahimi H (2009) Degradation of excess peroxisomes in mammalian liver cells by autophagy and other mechanisms. Histochem Cell Biol 131:455-458 doi:10.1007/s00418-0090564-6

Yu L, Chen Y, Tooze SA (2018) Autophagy pathway: Cellular and molecular mechanisms. Autophagy 14:207-215 doi:10.1080/15548627.2017.1378838

Yu L, McPhee CK, Zheng L, Mardones GA, Rong Y, Peng J, Mi N, Zhao Y, Liu Z, Wan F, Hailey DW, Oorschot V, Klumperman J, Baehrecke EH, Lenardo MJ (2010) Termination of autophagy and reformation of lysosomes regulated by mTOR. Nature 465:942-946 doi:10.1038/nature09076

Zhang J, Kim J, Alexander A, Cai S, Tripathi DN, Dere R, Tee AR, Tait-Mulder J, Di Nardo A, Han JM, Kwiatkowski E, Dunlop EA, Dodd KM, Folkerth RD, Faust PL, Kastan MB, Sahin M, Walker CL (2013) A tuberous sclerosis complex signalling node at the peroxisome regulates mTORC1 and autophagy in response to ROS. Nat Cell Biol 15:1186-1196 doi:10.1038/ncb2822

Zhang J, Tripathi DN, Jing J, Alexander A, Kim J, Powell RT, Dere R, Tait-Mulder J, Lee JH, Paull TT, Pandita RK, Charaka VK, Pandita TK, Kastan MB, Walker CL (2015) ATM functions at the peroxisome to induce pexophagy in response to ROS. Nat Cell Biol 17:1259-1269 doi:10.1038/ncb3230

Zientara-Rytter K, Ozeki K, Nazarko TY, Subramani S (2018) Pex3 and Atg37 compete to regulate the interaction between the pexophagy receptor, Atg30, and the Hrr25 kinase. Autophagy 14:368-384 doi:10.1080/15548627.2017.1413521

Zutphen T, Veenhuis M, van der Klei IJ (2008) Pex14 is the sole component of the peroxisomal translocon that is required for pexophagy. Autophagy 4:63-66 
Figure 1

a

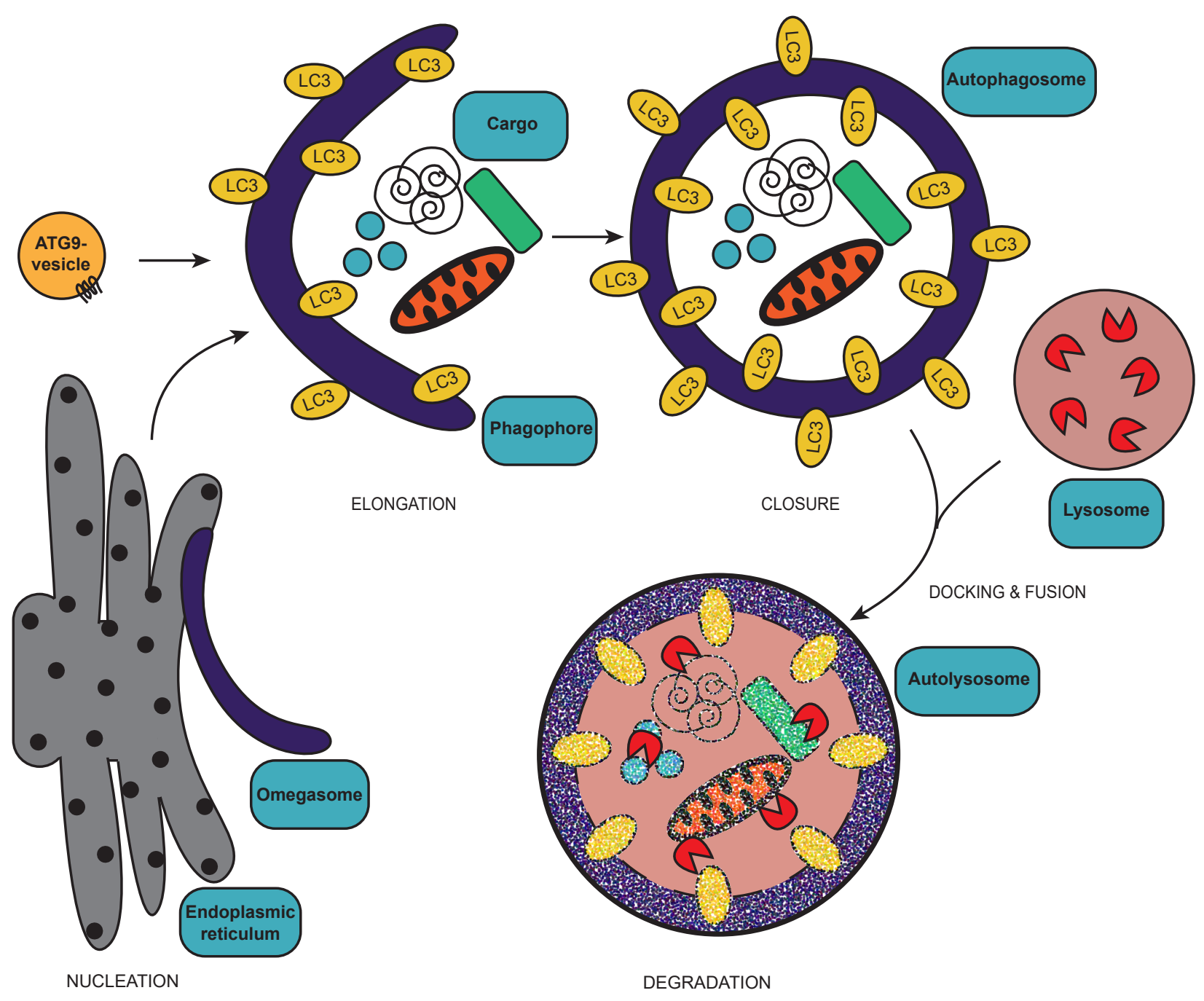

b

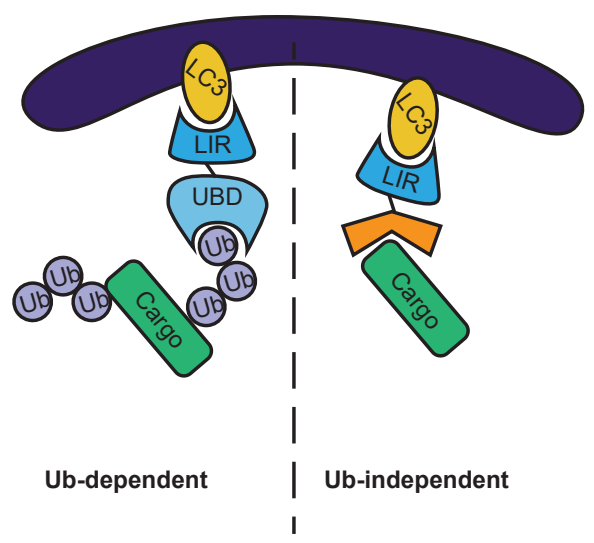




\section{Figure 2}

a

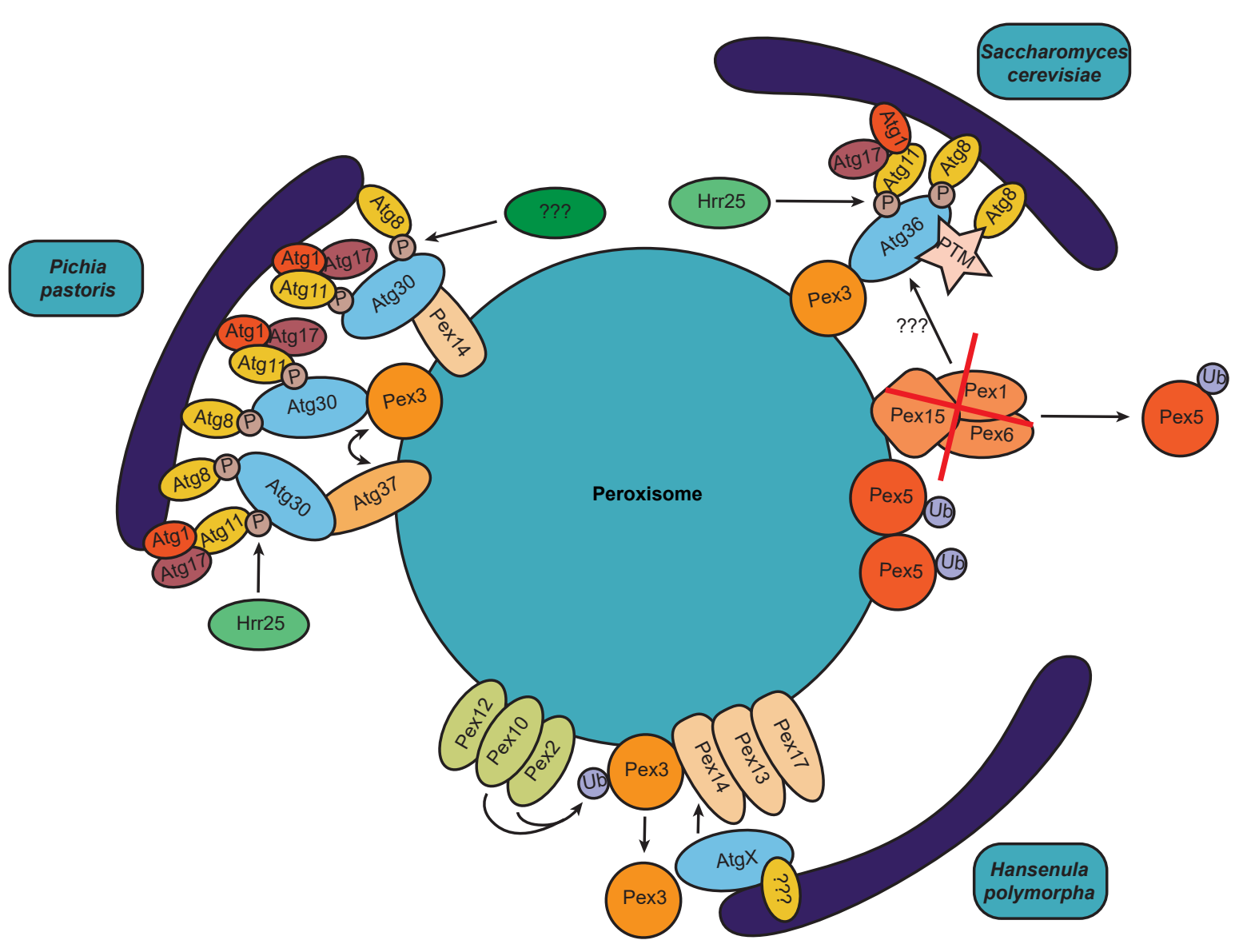

b

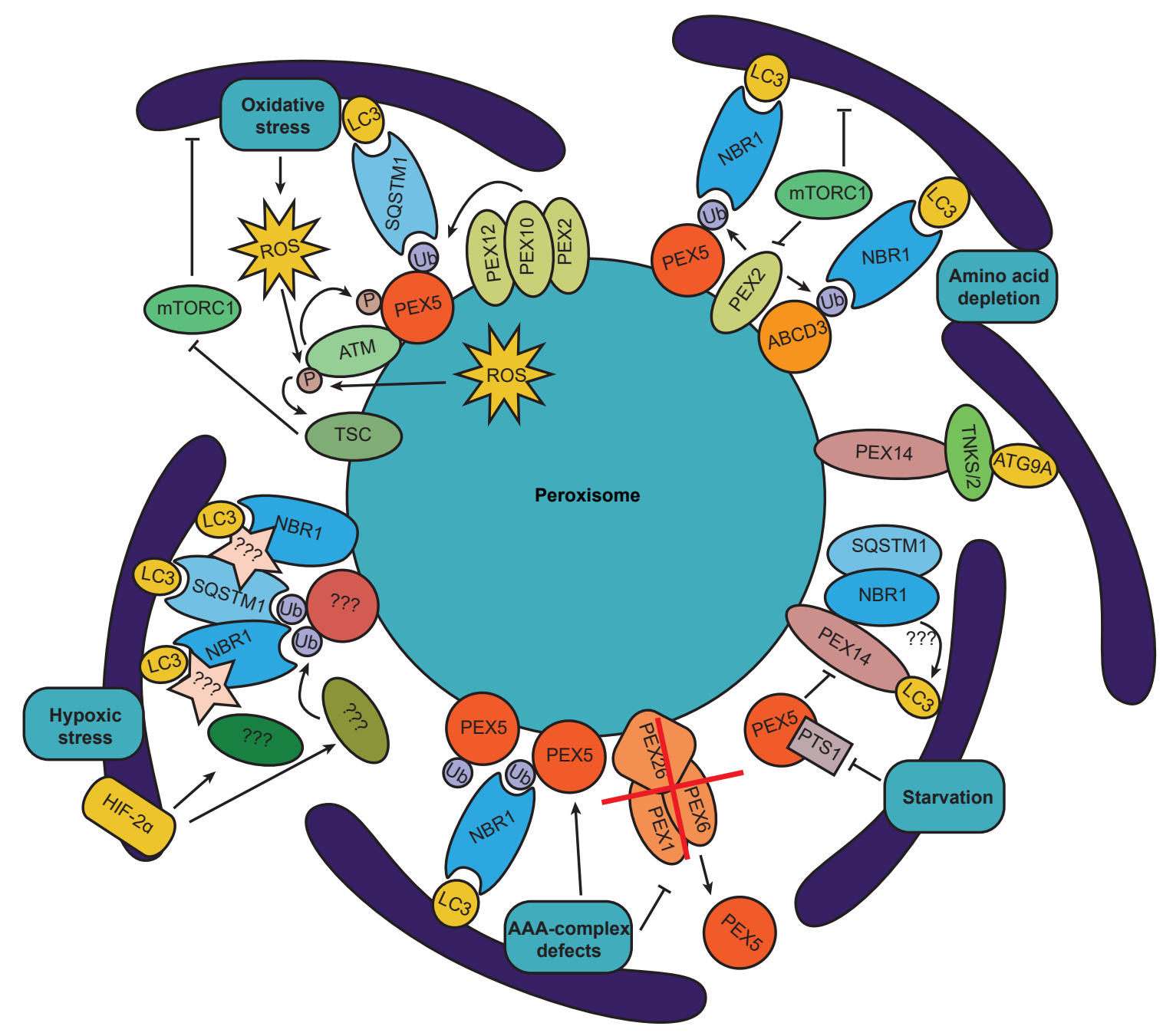

